\title{
Buried magnetic flux tubes in giant stars near the "Coronal Dividing Line"
}

\author{
V. Holzwarth and M. Schüssler
}

\author{
Max-Planck-Institut für Aeronomie, Max-Planck-Strasse 2, 37191 Katlenburg-Lindau, Germany \\ Received 2 May 2001 / Accepted 2 August 2001
}

\begin{abstract}
We apply the "solar paradigm" for stellar magnetic activity to the post-main-sequence evolution of stars in the mass range $1 M_{\odot} \leq M_{\star} \leq 3 M_{\odot}$. The model starts from a strong toroidal magnetic field generated by a dynamo working in the overshoot layer below the convection envelope. Once a critical field strength is exceeded, an undulatory (Parker-type) instability leads to rising flux loops. Upon emergence at the stellar surface, they form bipolar magnetic regions and large-scale coronal loops. By considering the stability, dynamics, and rise of magnetic flux tubes along evolutionary sequences of stellar models, we find that the flux loops become trapped in the stellar interior when the depth of convective envelope exceeds about $80 \%$ of the stellar radius. Trapping is caused by an increase of field line curvature at the loop summit, so that eventually the magnetic tension force dominates over the buoyancy force. The magnetic loops find a stable equilibrium configuration within the convection zone and do not emerge at the stellar surface. The transition from emerging to trapped flux tubes falls in the range of spectral types G7 to K0 for luminosity class III giants, which is close to the observed "coronal dividing line" in the Hertzsprung-Russell diagram. This result is remarkably stable within large ranges of stellar parameters (mass, rotation) and flux tube parameters (field strength, magnetic flux) and depends practically exclusively on the fractional radius of the stellar radiative core. We suggest that flux tube trapping is the cause for the strong decline of stellar X-ray emission across the "coronal dividing line".
\end{abstract}

Key words. stars: magnetic fields - stars: activity - stars: coronae - X-rays: stars - MHD

\section{Introduction}

Observations of stellar activity in various wavelength ranges indicate the existence of dividing lines in the cool half of the Hertzsprung-Russell (HR) diagram, which separate magnetically active and non-active (or less active) giants. The concept of dividing lines (DL) was introduced on the basis of early FUV observations by Linsky \& Haisch (1979) and of soft X-ray observations by Ayres et al. (1981) with the IUE and the Einstein observatory, respectively. These authors found a sharp division around mid $\mathrm{K}$ spectral type: hotter giants exhibit solar-like activity signatures with hot $\left(10^{5}-10^{6} \mathrm{~K}\right)$ gas indicating the existence of chromospheres, transition regions and coronae, while cooler giants only show chromospheric temperatures around $10^{4}$ K. Earlier, Reimers (1977) had found strong, cool outflows in giants cooler than mid $\mathrm{K}$ spectral type, which suggests a transition from hot coronae to massive, cool winds as a star crosses the DL region in the course of stellar evolution. Subsequent observations confirmed the striking decrease of coronal X-ray emission for giants and thus supported the DL concept (Maggio et al. 1990; Haisch et al. 1990). While these early observations were restricted to small samples and upper detection limits, the more

Send offprint requests to: V. Holzwarth, e-mail: holzwarth@linmpi.mpg.de sensitive ROSAT all-sky survey also indicated a clear dichotomy in the X-ray emission of cool giants (Haisch et al. 1991; Ayres et al. 1991). The sensitivity limits, however, still did not allow to exclude the existence of gas at coronal temperatures in cooler giants. Detections of X-ray emission in hybrid-spectrum stars (Reimers \& Schmitt 1992; Kashyap et al. 1994; Reimers et al. 1996), i.e., KII and GII-Ib stars showing signatures of both transition region line emission and absorption features indicating the existence of cool, massive stellar winds, led to the conclusion that the DL concept is not valid for bright giants and supergiants. More sensitive, pointed ROSAT observations confirmed a sharp drop of coronal emission around spectral type K3 for luminosity class III giants (Hünsch et al. 1996), but also revealed weak X-ray emission even on M giants (Hünsch et al. 1998). This leads to the conclusion that probably all giants show some level of X-ray emission if observed with sufficient sensitivity. On the other hand, all observations confirm the significant drop in X-ray emission and surface flux of ordinary giant stars around early K spectral type.

X-ray emission of cool stars requires the confinement of hot coronal matter in magnetic structures (coronal loops). Antiochos et al. (1986) suggested that the drop in $\mathrm{X}$-ray emission is caused by the instability of hot coronal loops as the gravitational scale height decreases during the 
evolutionary expansion of the star. Rosner et al. (1991, 1995) and Kashyap et al. (1994) conjectured a change of the dynamo mechanism as a giant evolves, which could lead to the transition of the atmospheric magnetic field structure from large coronal loops to smaller loops in between which a massive wind can escape along open magnetic field lines. Alternatively, it has been suggested that the coronal dividing line (CDL) originates from the juxtaposition in the HR diagram of populations of stars with different mass and therefore different (rotational) evolutionary history (Ayres et al. 1981, 1991; Reimers et al. 1996; Hünsch \& Schröder 1996).

In our study, we assume the existence of a dynamo mechanism operating in a rotational shear layer at the interface between the outer convection zone and the radiative core of a star. The amplified magnetic field is stored in the form of toroidal magnetic flux rings in the stably stratified overshoot region parallel to the equatorial plane, where it is subject to perturbations arising from penetrating convective gas motions. When the magnetic field strength exceeds a critical value, small displacements of the tube become unstable and form rising loops inside the convection zone, which produce upon emergence at the stellar surface magnetic activity (bipolar regions, starspots) and coronal loops (Schüssler et al. 1994). The quantitative elaboration of the flux tube model is consistent with various properties of solar active regions (Caligari et al. 1995) and with the emergence of polar spots on rapidly rotating single stars (Schüssler \& Solanki 1992; Schüssler et al. 1996; Granzer et al. 2000). In what follows, we extend this model to post-main-sequence stars (of low and moderate mass) near the base of the red giant branch (RGB). For ordinary giants - with a sufficiently small radiative core - near the CDL, we find the onset of a trapping mechanism which prevents flux tubes from reaching the stellar surface.

In Sect. 2 we explain the generation and the properties of the stellar models and the description of the overshoot region below the outer convection zone. Section 3 gives an exposition of the initial toroidal equilibrium, the linear stability analysis, and the non-linear numerical simulations. In Sect. 4 we show the results of our "standard" simulations and present a detailed parameter study. With the simple auxiliary model discussed in Sect. 5 we illustrate the physical reason for the onset of flux tube trapping. Section 6 gives a discussion of our results, including the consequences for the concept of the coronal dividing line, and Sect. 7 contains our conclusions.

\section{Stellar models}

\subsection{Evolutionary sequences}

For the generation of stellar models we used an updated version of the stellar evolution code of Kippenhahn et al. (1967). All calculations made use of the OPAL opacities (Iglesias \& Rogers 1996), extended with atomic and molecular opacities by Alexander \& Ferguson (1994).

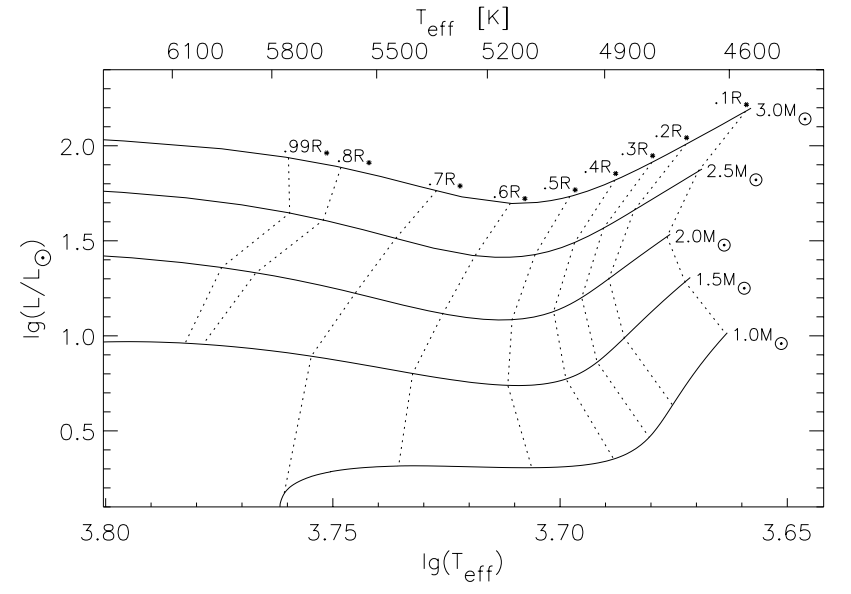

Fig. 1. Theoretical HR diagram with post-main-sequence evolutionary tracks (solid lines) of stars between $1 M_{\odot}$ and $3 M_{\odot}$, indicating the extension of their outer convection zone. Similar evolutionary stages, distinguished by the fractional size of the radiative core (core radius, $r_{\mathrm{rc}}$, in units of the stellar radius, $\left.R_{\star}\right)$, are connected with dotted lines.

The "Saha-like" equation of state was developed by Gautschy (priv. comm.) and the thermonuclear reaction rates were taken from Caughlan \& Fowler (1988). Local mixing length theory with a mixing length parameter $\alpha=1.67$ was used. The initial abundances were taken to be $X=0.703, Y=0.277$, and $Z=0.02$. Figure 1 shows a theoretical Hertzsprung-Russell diagram with the calculated post-main-sequence evolutionary tracks of stars with $1,1.5,2,2.5$, and $3 M_{\odot}$, respectively.

The dynamo scenario considered here (Schüssler et al. 1994) requires an outer stellar convection zone as an essential ingredient. While stars with mass $M \lesssim 1.3 M_{\odot}$ already possess an outer convection zone during their main-sequence phase, the higher-mass stars develop a convective envelope only when they have evolved off the mainsequence. In our evolutionary sequences, convection sets in for the $1.5 M_{\odot}$ star at $T_{\text {eff }} \simeq 6000 \mathrm{~K}$. This onset is shifted to somewhat lower temperatures for the highermass stars. From each sequence, we choose stellar models of similar evolutionary stage, so that the corresponding radii of the radiative core, $r_{\mathrm{rc}}$, lie in the range of $0.35 R_{\star} \gtrsim r_{\mathrm{rc}} \gtrsim 0.15 R_{\star}$, where $R_{\star}$ is the stellar radius.

\subsection{Convective overshoot}

The stellar models generated by the evolution code, calculated on basis of the local mixing length theory (MLT), consist of a weakly superadiabatic convection zone (superadiabaticity $\left.\delta_{\mathrm{cz}}=\nabla-\nabla_{\mathrm{ad}}>0\right)$ above a strongly subadiabatic radiative core $\left(\delta_{\mathrm{rc}}<0\right)$. We follow the approach of Granzer et al. (2000) and determine the convective overshoot region for the models a posteriori. This procedure, based upon the non-local MLT of Shaviv \& Salpeter (1973) and described in more detail by Pidatella \& Stix (1986) and Skaley \& Stix (1991), leads to gas elements which penetrate into the uppermost stable layer of 
Table 1. Properties of the stellar models used in this work. $R_{\star}, L_{\star}$ : stellar radius and luminosity (in solar units), $r_{\mathrm{bot}}$ : lower boundary of the overshoot region (in units of the solar and stellar radius, respectively), $d_{\mathrm{os}}$ : thickness of the overshoot layer, i.e., the region of downward directed convective energy flux (in units of the solar radius, stellar radius, and as fraction of the local pressure scale height, $H_{\mathrm{p}}$ ), $\delta$ : superadiabaticity in the middle of the overshoot layer (at "standard" equilibrium depth $r_{\mathrm{eq}}$ ).

\begin{tabular}{|c|c|c|c|c|c|c|c|c|c|c|c|}
\hline \multirow{2}{*}{$\begin{array}{c}M \\
{\left[M_{\odot}\right]}\end{array}$} & \multirow[t]{2}{*}{ Nos. } & \multirow{2}{*}{$\begin{array}{l}\text { age } \\
{[\mathrm{yr}]}\end{array}$} & \multirow{2}{*}{$\begin{array}{r}L_{\star} \\
{\left[L_{\odot}\right]} \\
\end{array}$} & \multirow{2}{*}{$\begin{array}{l}T_{\text {eff }} \\
{[\mathrm{K}]} \\
\end{array}$} & \multirow{2}{*}{$\begin{array}{r}R_{\star} \\
{\left[R_{\odot}\right]}\end{array}$} & \multicolumn{2}{|c|}{$r_{\text {bot }}$} & \multicolumn{3}{|c|}{$d_{\mathrm{os}}$} & \multirow{2}{*}{$\begin{array}{c}\delta\left(r_{\mathrm{eq}}\right) \\
{\left[10^{-6}\right]}\end{array}$} \\
\hline & & & & & & {$\left[R_{\odot}\right]$} & {$\left[R_{\star}\right]$} & {$\left[\begin{array}{ll}10^{-2} & R_{\odot}\end{array}\right]$} & {$\left[\begin{array}{ll}10^{-2} & R_{\star}\end{array}\right]$} & {$\left[H_{\mathrm{p}}\right]$} & \\
\hline \multirow[t]{8}{*}{1.0} & 465 & $1.193 \mathrm{E} 10$ & 2.35 & 4854 & 2.17 & 0.75 & 0.34 & 7.6 & 3.5 & 0.27 & -1.0 \\
\hline & 480 & $1.198 \mathrm{E} 10$ & 2.58 & 4820 & 2.31 & 0.71 & 0.31 & 8.0 & 3.5 & 0.26 & -1.1 \\
\hline & 495 & $1.203 \mathrm{E} 10$ & 2.83 & 4798 & 2.44 & 0.67 & 0.28 & 8.2 & 3.4 & 0.26 & -1.2 \\
\hline & 510 & $1.208 \mathrm{E} 10$ & 3.07 & 4782 & 2.56 & 0.64 & 0.25 & 8.3 & 3.2 & 0.25 & -1.3 \\
\hline & 525 & $1.211 \mathrm{E} 10$ & 3.33 & 4769 & 2.68 & 0.62 & 0.23 & 8.2 & 3.1 & 0.25 & -1.5 \\
\hline & 540 & $1.214 \mathrm{E} 10$ & 3.59 & 4759 & 2.79 & 0.59 & 0.21 & 8.2 & 2.9 & 0.25 & -1.6 \\
\hline & 585 & $1.221 \mathrm{E} 10$ & 4.30 & 4735 & 3.09 & 0.54 & 0.18 & 7.9 & 2.5 & 0.24 & -2.3 \\
\hline & 675 & $1.232 \mathrm{E} 10$ & 6.02 & 4690 & 3.72 & 0.48 & 0.13 & 7.1 & 1.9 & 0.24 & -3.9 \\
\hline \multirow[t]{5}{*}{1.5} & 480 & $2.447 \mathrm{E} 9$ & 6.42 & 4958 & 3.44 & 1.09 & 0.32 & 13 & 3.8 & 0.27 & -1.8 \\
\hline & 510 & $2.478 \mathrm{E} 9$ & 7.25 & 4919 & 3.71 & 0.98 & 0.26 & 13 & 3.6 & 0.25 & -2.2 \\
\hline & 570 & $2.518 \mathrm{E} 9$ & 8.67 & 4877 & 4.13 & 0.84 & 0.20 & 13 & 3.2 & 0.24 & -3.1 \\
\hline & 600 & $2.535 \mathrm{E} 9$ & 9.44 & 4858 & 4.35 & 0.78 & 0.18 & 13 & 3.0 & 0.24 & -3.7 \\
\hline & 660 & 2.563E9 & 11.05 & 4825 & 4.76 & 0.69 & 0.15 & 12 & 2.5 & 0.24 & -5.1 \\
\hline \multirow[t]{6}{*}{2.0} & 374 & $9.161 \mathrm{E} 8$ & 14.68 & 4986 & 5.14 & 1.56 & 0.30 & 20 & 3.9 & 0.26 & -3.2 \\
\hline & 376 & $9.171 \mathrm{E} 8$ & 15.64 & 4963 & 5.36 & 1.45 & 0.27 & 20 & 3.8 & 0.25 & -3.5 \\
\hline & 378 & $9.179 \mathrm{E} 8$ & 16.47 & 4946 & 5.54 & 1.35 & 0.24 & 20 & 3.6 & 0.25 & -3.9 \\
\hline & 382 & $9.195 \mathrm{E} 8$ & 18.08 & 4918 & 5.87 & 1.20 & 0.20 & 20 & 3.3 & 0.24 & -5.3 \\
\hline & 386 & $9.210 \mathrm{E} 8$ & 19.62 & 4895 & 6.17 & 1.08 & 0.18 & 19 & 3.1 & 0.24 & -6.8 \\
\hline & 388 & $9.215 \mathrm{E} 8$ & 20.23 & 4886 & 6.29 & 1.04 & 0.17 & 19 & 3.0 & 0.24 & -7.3 \\
\hline \multirow[t]{6}{*}{2.5} & 646 & $4.784 \mathrm{E} 8$ & 33.49 & 4940 & 7.91 & 2.49 & 0.32 & 29 & 3.7 & 0.25 & -5.7 \\
\hline & 647 & $4.787 \mathrm{E} 8$ & 36.30 & 4908 & 8.35 & 2.26 & 0.27 & 29 & 3.5 & 0.24 & -6.8 \\
\hline & 649 & $4.791 \mathrm{E} 8$ & 41.31 & 4862 & 9.07 & 1.89 & 0.21 & 30 & 3.3 & 0.24 & -9.2 \\
\hline & 650 & $4.792 \mathrm{E} 8$ & 43.60 & 4844 & 9.39 & 1.75 & 0.19 & 30 & 3.2 & 0.25 & -10.5 \\
\hline & 653 & $4.795 \mathrm{E} 8$ & 47.17 & 4818 & 9.88 & 1.58 & 0.16 & 29 & 2.9 & 0.25 & -13.0 \\
\hline & 655 & $4.797 \mathrm{E} 8$ & 49.44 & 4802 & 10.18 & 1.50 & 0.15 & 28 & 2.8 & 0.25 & -14.7 \\
\hline \multirow[t]{5}{*}{3.0} & 466 & $2.913 \mathrm{E} 8$ & 75.28 & 4817 & 12.48 & 3.75 & 0.30 & 47 & 3.8 & 0.26 & -13.4 \\
\hline & 468 & $2.915 \mathrm{E} 8$ & 89.95 & 4748 & 14.04 & 3.08 & 0.22 & 46 & 3.3 & 0.24 & -15.6 \\
\hline & 469 & $2.916 \mathrm{E} 8$ & 96.59 & 4721 & 14.71 & 2.78 & 0.19 & 45 & 3.1 & 0.24 & -16.9 \\
\hline & 470 & $2.917 \mathrm{E} 8$ & 103.37 & 4697 & 15.38 & 2.51 & 0.16 & 43 & 2.8 & 0.24 & -20.8 \\
\hline & 471 & $2.918 \mathrm{E} 8$ & 110.38 & 4673 & 16.06 & 2.30 & 0.14 & 40 & 2.5 & 0.24 & -25.5 \\
\hline
\end{tabular}

the radiative core and thus extend the original convection zone; the overshoot region is characterized by a downward directed convective energy flux. The lower boundary of the overshoot region, $r_{\mathrm{bot}}\left(<r_{\mathrm{rc}}\right)$, is determined by the vanishing of convective motion. Table 1 gives a summary of various parameters for the stellar models which we have considered in our study of flux tube dynamics.

\section{Model assumptions and procedure}

Throughout our investigation we used the thin-flux-tube approximation (Spruit 1981) for the description of the magnetic field and treated the stellar interior as an ideal plasma.

\subsection{Magnetic equilibrium and stability}

Our considerations start with the assumption of an isolated, toroidal magnetic flux tube (i.e., a flux ring) embedded in the stably stratified overshoot region below the outer convection zone. The plane of the tube is perpendicular to the rotation axis of the star. The flux ring is in stationary mechanical equilibrium, i.e., the tube is nonbuoyant and the magnetic tension force is compensated by the Coriolis force caused by a longitudinal flow inside the tube (Moreno-Insertis et al. 1992). Convective motions in the overshoot region perturb the flux tube, which responds with a discrete spectrum of eigenmodes. Once a critical magnetic field strength is exceeded, an undulatory (Parker-type) instability sets in and small displacements continue to grow (Spruit \& van Ballegooijen 1982; Ferriz-Mas \& Schüssler 1993, 1995). The stability properties depend on the magnetic field strength, the stratification into which the tube is embedded, its radius of curvature, and on the rotation rate of the star. In our simulations, the equilibrium position of flux tubes (the radius of equilibrium, $r_{\text {eq }}$ ) was taken to be the middle of the overshoot region. Table 1 summarizes some characteristic values at the equilibrium position for the stellar models considered. For our "standard" simulations we assumed rigid rotation with solar rotation rate, $\Omega_{\odot}=2.6 \times 10^{-6} \mathrm{~s}^{-1}$. Unless the differential rotation is much stronger than in the case of the sun, its influence on the stability behaviour is only marginal (Ferriz-Mas \& Schüssler 1995). Other 
possible external flows (e.g., meridional circulation) are not considered.

By the choice of the stellar model, rotation rate and equilibrium radius, all quantities outside the flux ring are determined. Stability properties then only depend on the latitude, $\lambda$, and magnetic field strength, $B$, of the equilibrium flux tube. The characteristic growth times, $\tau$, of unstable eigenmodes with azimuthal wave number $m$ are determined by a linear stability analysis (FerrizMas \& Schüssler 1995), assuming adiabatic perturbations in the limit of small magnetic field strengths (i.e., $\beta=8 \pi p / B^{2} \gg 1$, where $p$ is the gas pressure). We consider wave numbers $m \leq 3$, because in the overshoot region the magnetic tension force typically prevents instabilities with higher wave numbers. For our "standard" simulations we choose a growth time of $\tau=100 \mathrm{~d}$. Figure 2 shows lines of constant growth time in the $(B, \lambda)$-plane for each stellar model and evolutionary stage listed in Table 1.

From the equator up to mid-latitudes the critical magnetic field strength, $B_{100}$, is nearly constant along an isoline. This region belongs to the buoyancy-driven, undulatory (Parker-type) instability. Toward higher latitudes, $B_{100}$ increases significantly: the shrinking radius of curvature of an equilibrium flux tube induces a growing tension force, which stabilizes perturbations perpendicular to the axis of rotation. At very high latitudes, the "poleward slip" instability leads to a decrease of $B_{100}$. Within an evolutionary sequence, this isoline pattern is typically shifted toward higher magnetic field strength as the star ages. However, the increase of field strength with age becomes smaller for stars with higher mass. For the $2.5 M_{\odot}$ star there is only a marginal increase of $B_{100}$ while for the $3 M_{\odot}$ sequence the shift reverses, i.e., flux rings in older models become unstable at lower field strengths. This tendency is enhanced by the fact that for higher-mass stars the instability generally sets in at lower field strengths. Up to high latitudes, the instability with azimuthal wave number $m=1$ has the largest growth rate. Only near the pole the $m=0$ ("poleward slip") instability dominates.

\subsection{Numerical simulations}

We follow numerically the non-linear, adiabatic evolution of an unstable flux tube through the convection zone, using a code which is described in detail by Caligari et al. (1995). An initial disturbance, consisting of a superposition of small displacements with azimuthal wave numbers $m=0 \ldots 5$ and total amplitude of less than one percent of the local pressure scale height, is applied to flux rings at equilibrium latitudes of $\lambda=10^{\circ}, 30^{\circ}, 50^{\circ}$ and $70^{\circ}$, respectively. The critical magnetic field strength, $B_{100}$, is taken from the corresponding isoline in the $(B, \lambda)$-plane (see Fig. 2). After some time, the fastest growing eigenmode (typically $m=1$ ) becomes dominant and causes the formation of a rising loop. The back reaction of the stellar environment on the moving tube is taken into account through the aerodynamic drag force, which depends on

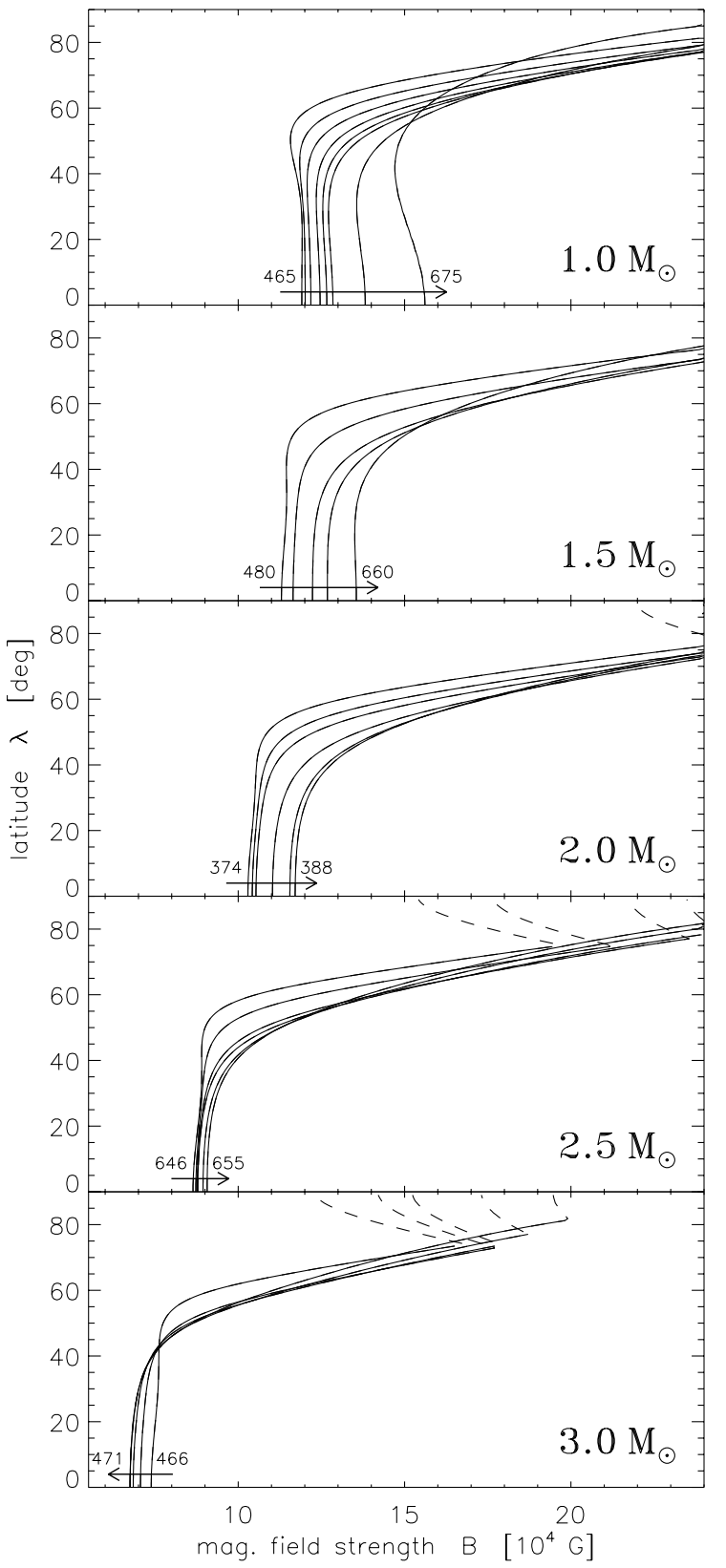

Fig. 2. Lines of constant growth time (100 d) for "standard" equilibrium flux tubes (described in Sect. 3.1). Solid and dashed lines correspond to azimuthal wave numbers $m=1$ and $m=0$, respectively. Each line corresponds to one of the stellar models in Table 1 (arrows indicate the chronological sequence of evolutionary stages).

the radius of a tube element and its normal velocity relative to the external medium. The initial radius of the flux tube is set to $1000 \mathrm{~km}$. Due to the conservation of magnetic flux and the lateral pressure balance between the exterior and interior of a tube $\left(p_{\text {ext }}=p_{\text {int }}+B^{2} / 8 \pi\right)$, a rising segment of a tube expands. The evolution can be followed up to layers near the stellar surface $\left(\sim 0.98 R_{\star}\right)$, where the thin-flux-tube approximation breaks down. 


\section{Evolution of magnetic flux tubes}

\section{1. "Standard" cases}

Our simulations reveal two different characteristic behaviours of rising magnetic flux tubes in giant stars. We describe these in detail for the case of a $1 M_{\odot}$ star.

Emerging flux tubes: Figures $3 \mathrm{a}-\mathrm{b}$ illustrate the evolution of a rising flux tube in a star of 12.14 Gyr age (No. 540 in Table 1). The initial equilibrium latitude is $10^{\circ}$. An unstable loop forms, enters the convection zone, and continues to rise and to accelerate until it reaches the surface. During the rise, the tube elements remaining in the overshoot region sink further down until their increasing buoyancy compensates the sum of the radial components of the magnetic tension and Coriolis forces. These bottom elements, located close to the top of the radiative core, form a kind of anchor for the rising flux loop. On the other hand, the latitudinal component of the tension force cannot be balanced by buoyancy. Since this component is pointing toward higher latitudes, the anchor slips poleward on top of the radiative core and crosses the pole (in this example) after 1130 days. Since the anchored segment remains in the overshoot region, the latitudinal component of the tension force now leads to an equatorward drift of the bottom elements until the top of the tube reaches the surface and the simulation has to be stopped. Figure 3c shows the position of the lowest and highest tube element (in terms of distance from the stellar center) as a function of time. The azimuthal drift of the rising segment in the direction opposite to stellar rotation reflects the propagating wave character of the instability. The whole evolution takes about 3.6 years.

Trapped flux tubes: Figures $3 \mathrm{~d}-\mathrm{f}$ show the evolution of a flux tube in a star of 12.21 Gyr age (No. 585). The initial evolution is quite similar to the previous case, i.e., the rising top segment is accelerated upwards and the anchored part of the tube slips toward the pole of the radiative core and crosses it. However, after the pole traversal, the rise of the loop slows down until it stops inside the convection zone well below the surface. In the following time, the whole flux tube slowly drifts toward the equator until it finally finds a new equilibrium in the equatorial plane of the star. In contrast to the original equilibrium, the flux tube no longer encloses the radiative core. The evolution from the initial to the final equilibrium takes about 7 years.

The simulations show that the transition from emerging to trapped flux tubes takes place when the relative radius of the initial flux ring, $\mathcal{R}$, satisfies

$\mathcal{R}=\frac{R_{\mathrm{eq}}}{R_{\star}}=\frac{r_{\mathrm{eq}}}{R_{\star}} \cos \lambda_{\mathrm{eq}} \lesssim 0.17 \ldots 0.2$,

where $R_{\text {eq }}$ is the radius of curvature of the initial equilibrium flux ring. For larger values of $\mathcal{R}$, flux tubes emerge at the surface, whereas for values below this threshold the rise is stopped and the tube becomes trapped deep inside the convection zone. As the star evolves toward the RGB, its radiative core shrinks $\left(r_{\text {bot }} \downarrow\right)$ while its convective envelope expands $\left(R_{\star} \uparrow\right)$. Consequently, for a fixed initial latitude, $\lambda_{\text {eq }}$, of the flux tube, the relative radius $\mathcal{R} \propto r_{\text {bot }} / R_{\star}$ decreases (see Eq. (1) with $r_{\text {eq }} \sim r_{\text {bot }}$ ). Since $\mathcal{R} \propto \cos \lambda_{\text {eq }}$, flux tubes at high latitudes are trapped first while lowlatitude loops still emerge. When $\mathcal{R}$ falls short of the critical value for all latitudes, flux emergence is completely inhibited. As an example, Fig. 4 shows the evolution of the radial position of the loop summits for model No. 540, depending on the initial latitude of the flux ring. While tubes with $\lambda_{\text {eq }} \lesssim 33^{\circ}$ erupt, those with larger initial latitudes are trapped.

The transition from erupting to trapped magnetic flux tubes takes place in all evolutionary sequences considered. Figure 5 shows a theoretical HR diagram with all stellar models investigated. The symbols indicate the different behaviours of magnetic flux tubes.

\subsection{Parameter dependence}

For the sake of comparability, all simulations discussed above were based upon the same "standard" assumptions: solar rotation rate, 100 days growth time of the linear instability, initial flux tube radius of $1000 \mathrm{~km}$, equilibrium flux ring located in the middle of the overshoot region. To investigate the dependence of the results on these assumptions, we successively varied one of the parameters over a reasonable range of values while keeping the other specifications unchanged.

Rotation rate: Due to magnetic braking and expansion, the rotation of evolving post-main-sequence single stars usually slows down considerably. On the other hand, the rotation rate of tidally coupled giant stars in close binary systems (e.g., RS CVn systems) can exceed the solar value by a factor of 10 . Therefore, we have considered rotation rates in the range of $0.1 \Omega_{\odot} \leq \Omega \leq 10 \Omega_{\odot}$. In general, we find no significant influence of the rotation rate on the behaviour of flux tubes compared with our standard simulations. However, at high rotation rates trapped flux tubes tend to remain at high latitudes and do not sink to the equator. They find a dynamical state, where both the anchored part (in the overshoot region at high latitudes) and the loop summit (deep inside the convection zone) precess around the rotation axis of the star. Such flux tubes do not emerge and therefore are trapped, although they do not reach the "standard" equilibrium in the equatorial plane as described in the former section. For small rotation rates, we find that the transition latitude which divides erupting from trapped flux tubes is shifted somewhat toward higher latitudes.

Growth time/initial magnetic field strength: The linear stability analysis shows that the critical magnetic field strength for the occurrence of an instability is only about $15 \%$ below $B_{100}$ (corresponding to $100 \mathrm{~d}$ growth time). Larger linear growth times (i.e., toward the edge of the instability region) would therefore only lead to a minor decrease of the initial magnetic field strength and to a slight 

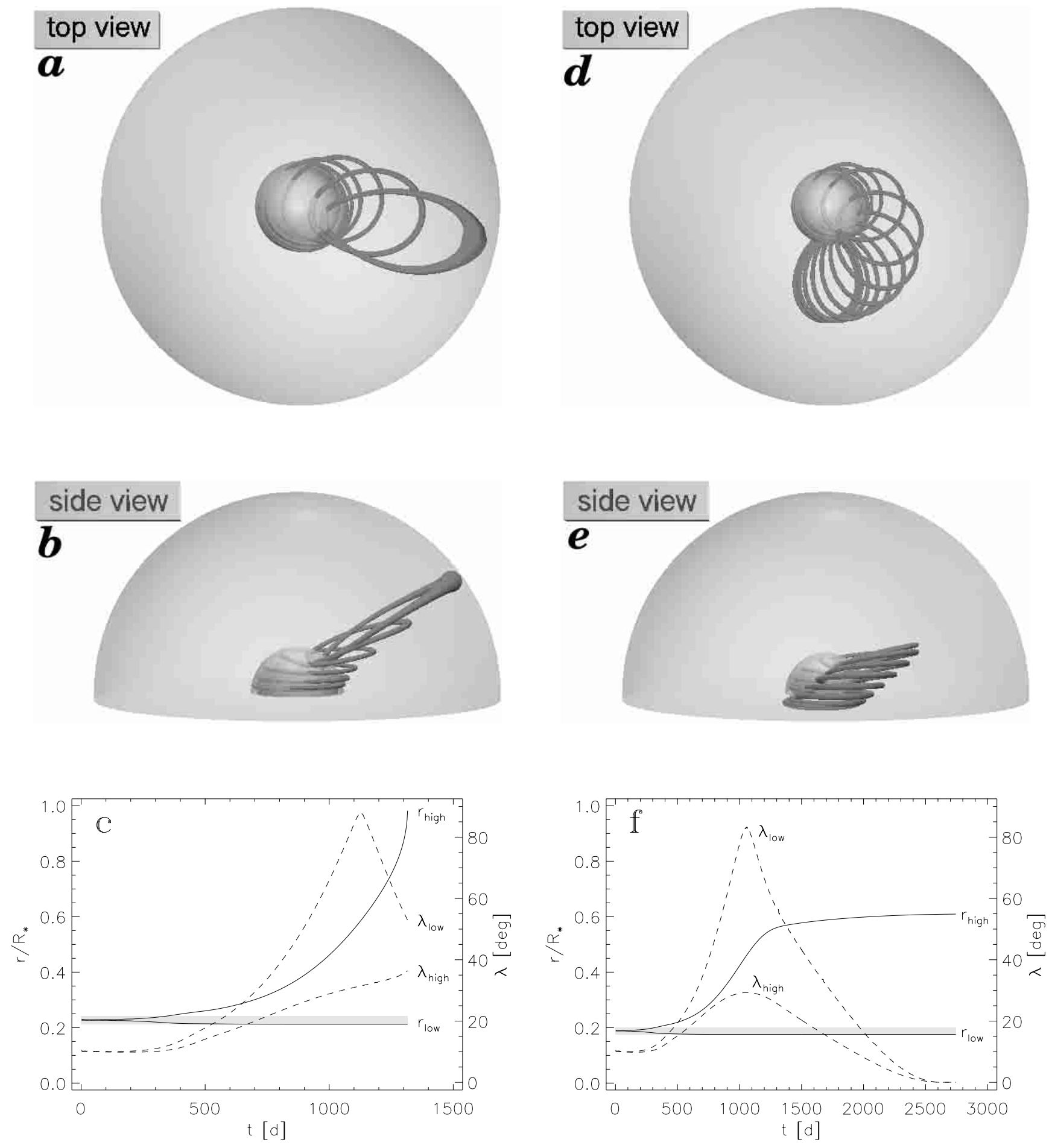

Fig. 3. Examples of emerging and trapped flux tubes. Panels a) and b) show snapshots of the evolution of an emerging flux tube from two different points of view. The innermost hemisphere represents the radiative core of the star and the (semi-transparent) thin layer above it the overshoot region (stellar model No. 540, $r_{\text {bot }}=0.21 R_{\star}, d_{\text {os }}=2.9 \times 10^{-2} R_{\star}$ ). The rotation of the star is in counter-clockwise direction. Note the expansion of the top element close to the stellar surface. The diameter of the tubes are magnified by a factor of 10 for better visibility. Panel c) gives the radial (solid lines, left scale) and latitudinal (dashed lines, right scale) position of the highest and lowest tube element, respectively, with the peak in the $\lambda_{\text {low-curve indicating the }}$ moment of pole crossing. The grey shaded, horizontal stripe depicts the extent of the overshoot region. Panels $\mathbf{d})-\mathbf{f})$ give the same illustrations for a trapped flux tube (stellar model No. 585, $r_{\mathrm{bot}}=0.18 R_{\star}, d_{\mathrm{os}}=2.5 \times 10^{-2} R_{\star}$ ). After pole crossing took place, the further rise of the top element slows down significantly and the whole tube drifts toward the equatorial plane. 


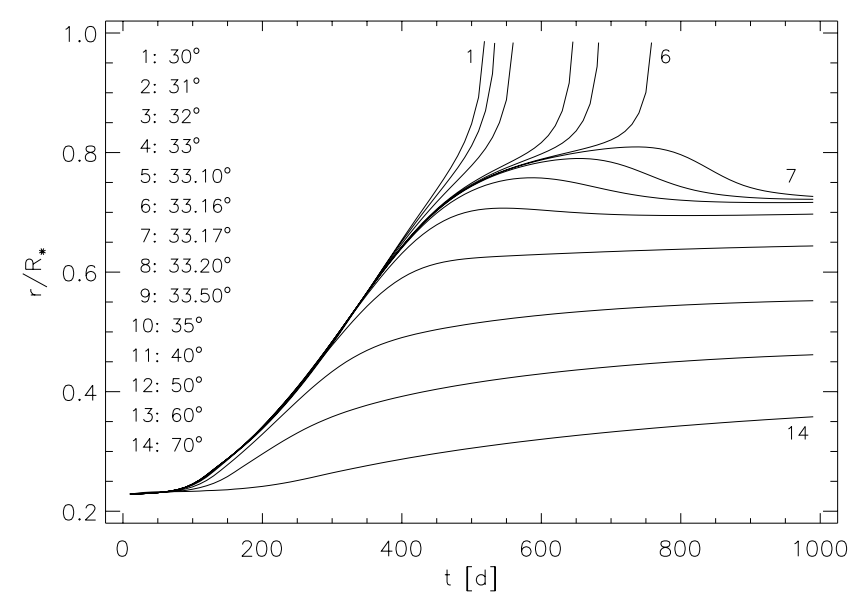

Fig. 4. Radial position of the tube summit in the $1 M_{\odot}$ star of 12.14 Gyr age (No. 540 in Table 1). The curves correspond to flux tubes with different initial equilibrium latitudes, $\lambda_{\text {eq. }}$. The transition between erupting and trapped flux tubes takes place at $\lambda_{\mathrm{eq}} \simeq 33.16^{\circ}$. The initial magnetic field strength of all flux tubes was set to $B=2 \times 10^{5} \mathrm{G}$ so that tubes starting at high latitudes could also be included in the comparison.

modification of the standard equilibrium. On the other hand, a decrease of $\tau$ leads to significantly higher magnetic field strengths. We performed additional simulations with linear growth times down to about 16 days, which result in magnetic field strengths typically twice as large as the corresponding "standard" values. We find that higher initial field strengths significantly accelerate the time evolution of both erupting and trapped flux tubes, but do not alter their final state; in particular, the transition latitude is only affected marginally.

Magnetic flux: The magnetic flux, $\Phi$, is determined by the tube diameter and the magnetic field strength. With the assumption of an initial tube radius of $1000 \mathrm{~km}$ and field strengths determined by the linear stability, our "standard" simulations cover a range of $\Phi \sim 10^{21} \ldots 10^{22} \mathrm{Mx}$, which corresponds to large active regions on the sun. While starspots on active giants can be much larger than that (see e.g., Strassmeier 1999), these observations cannot distinguish between the emergence of a small number of large flux tubes and a large number of smaller ones. Since the overshoot regions of giant stars are very wide (see Table 1), neither larger tubes nor a greater number of smaller tubes pose a problem to the basic assumption of flux storage in the form of isolated flux tubes. The size of a tube affects the aerodynamic drag force. However, compared with the strength of the other forces (e.g., magnetic tension and buoyancy), the relative influence of the drag force decreases for increasing tube radius and the drag force eventually becomes negligible. We have repeated a number of simulations with the standard field strengths and initial flux tube radii of $500 \mathrm{~km}$ and $2000 \mathrm{~km}$, respectively. The results show that the considered range of tube radii is already in the regime where the back reaction of

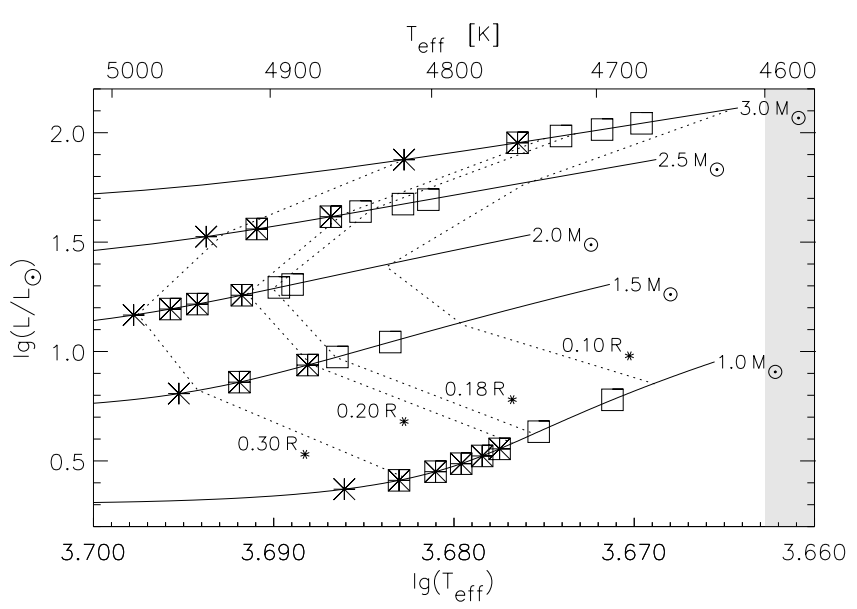

Fig. 5. Evolutionary tracks (solid lines) near the base of the RGB. Dotted lines connect similar evolutionary stages which are distinguished by the relative size of the radiative core. Asterisks and squares denote stellar models with emerging and trapped flux tubes, respectively. Both symbols are over-plotted if high-latitude flux tubes are trapped whereas low-latitude tubes emerge. The shaded stripe on the right indicates the region where various authors have located the Coronal Dividing Line.

the external medium and, therefore, the amount of magnetic flux has no effect on the final state of the evolution. Initial depth of equilibrium: The stability properties of a flux ring depend sensitively on the value of the superadiabaticity, $\delta$, which varies across the overshoot layer by a factor of about 100 . We have carried out simulations with the equilibrium flux ring located at the top of the overshoot region and about $2000 \mathrm{~km}$ above its lower boundary, respectively. The starting position at the more stable region close to the radiative core requires substantially larger magnetic field strengths for the onset of instability, which leads to a significant acceleration of the flux tube evolution. However, the final stages hardly differ from our standard simulations. Although this is also true for most flux tubes starting from the top of the overshoot layer, sometimes we found a different evolutionary behaviour: flux rings starting at very high latitudes detach from the overshoot region due to the poleward slip instability and drift upward along the rotation axis until they emerge near the stellar pole. Such behaviour was also found for flux tubes in fast-rotating T-Tauri stars (Granzer et al. 2000).

In summary, we find that variations of the parameters within reasonable ranges have only minor influence on the fate of rising flux tubes. The decision whether a tube emerges at the surface or becomes buried inside the convection zone depends nearly exclusively on the initial relative radius of curvature, $\mathcal{R}$. 


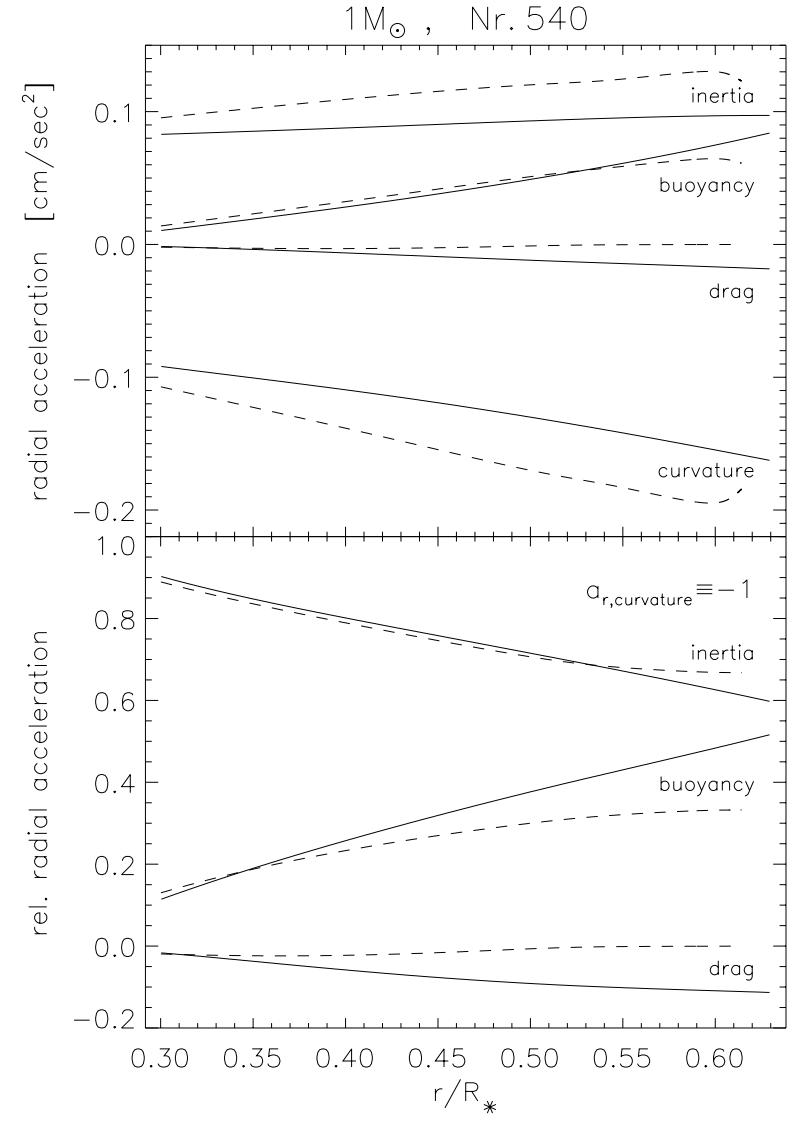

Fig. 6. Contributions of various forces to the radial acceleration of the uppermost tube element of an erupting (solid lines) and a trapped flux tube (dashed lines), respectively. The lower panel gives the relative contribution normalized to the magnetic tension acceleration (set to -1 ). The initial magnetic field strength for both tubes is $B=1.28 \times 10^{5} \mathrm{G}$. The final equilibrium height of the trapped tube in the equatorial plane is about .61 $R_{\star}$.

\section{Why do flux tubes become trapped?}

\subsection{Forces breakdown}

In order to find the reason for their different behaviour, we compare an erupting flux tube starting at $10^{\circ}$ latitude of the $1 M_{\odot}$ star (No. 540, the case shown in Figs. 3a-c), and a trapped flux tube starting at $40^{\circ}$ latitude of the same stellar model. Both tubes start in the middle of the overshoot region with the same magnetic field strength; they only differ in latitude (i.e., radius of curvature) and in the internal flow velocity required to balance the magnetic tension force. The transition between erupting and trapped flux tubes takes place at about $29.5^{\circ}$ latitude. Figure 6 shows the contributions of the various forces to the radial acceleration of the uppermost tube element as a function of its radial position inside the star.

The drag force opposes the rise of the summit and reflects its ascending velocity. Since it is a consequence of the motion and not its origin, it can only modify the ascent but not considerably alter the final state and is, therefore, of minor importance. The inertial acceleration is mainly due to the Coriolis force. Its relative contribution to the rise of the top element of both erupting and trapped flux tubes is similar and so we do not consider this force to have a decisive influence. The buoyancy force is the driver of an unstable, rising loop. While it is somewhat stronger for the trapped tube in the lower part of the convection zone, it increases slower with height and falls short of the value for the erupting flux tube in the middle of the convection zone. The growth of the inward directed magnetic tension force is due to the large increase of curvature at the top of the loop, which overcompensates the drop of magnetic field strength during the rise.

This relation indicates that the specific variation of both buoyancy force and magnetic tension force is responsible for the trapping of magnetic flux tubes in giant stars. The slower increase of buoyancy (i.e., density decrease) with height in the summit of tubes starting at higher latitudes is due to a weakening of the net downflow of matter compared to the rise of tubes starting at lower latitudes. For the tension force, the decline of the magnetic field strength with height (due to the expansion of the loop summit) is more than compensated by the strong increase of curvature. Therefore, the drop of the outward directed buoyancy force is accompanied by a still increasing, inward directed magnetic tension force, which results in a deceleration of the summit of high latitude tubes. Equilibrium flux tubes at higher latitudes start their evolution already with a larger curvature and thus are much more susceptible to this decelerating effect. Above a critical equilibrium latitude, this influence stalls the summit of the tubes and terminates their rise.

\subsection{Polytropic layer model}

In order to illustrate the circumstances under which trapping occurs, we propose a simplified model which nevertheless contains essential elements of the original problem.

We consider a hydrostatic stratification without rotation. With the assumption of a power law for the gravitational acceleration, the condition for hydrostatic equilibrium reads

$\frac{\mathrm{d} p}{\mathrm{~d} r}=-\rho g=-\rho(r) g_{0}\left(\frac{r_{0}}{r}\right)^{\sigma}$

The index " 0 " refers to values at a reference radius, $r_{0}$. Gas pressure, $p$, and density, $\rho$, are assumed to follow the polytropic relation

$p=K \rho^{\gamma^{*}}$

where $K$ and $\gamma^{*}$ are the polytropic constant and exponent, respectively. The combination of Eqs. (2) and (3) leads to the equation

$-g_{0}\left(\frac{r_{0}}{r}\right)^{\sigma} \mathrm{d} r=\gamma^{*} K \rho^{\left(\gamma^{*}-2\right)} \mathrm{d} \rho$, 


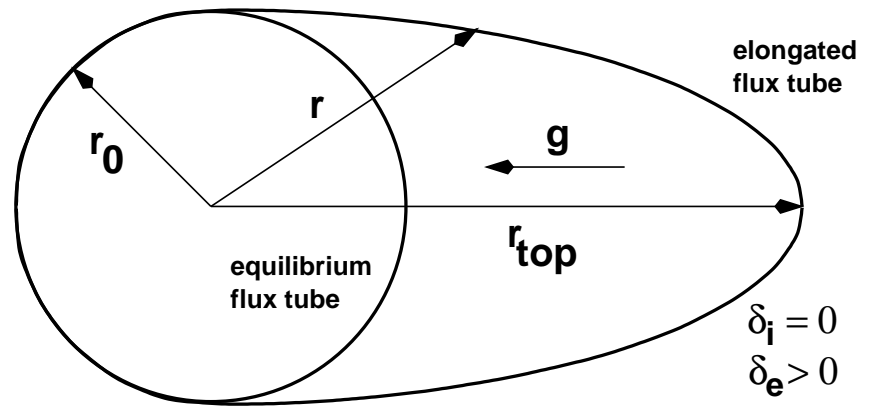

Fig. 7. Sketch of the flux tube in the polytropic layer model. A toroidal equilibrium flux ring with initial radius $r_{0}$ is stretched adiabatically and quasi-statically in the polytropic layer, with the uppermost tube element at $r_{\text {top }}$. The hydrostatic stratification outside the tube is superadiabatic.

which is integrated from radius $r_{0}$ to $r$. With the usual definition of the logarithmic temperature gradient,

$\nabla^{*}=\left(\frac{\mathrm{d} \ln T}{\mathrm{~d} \ln p}\right)=\frac{\gamma^{*}-1}{\gamma^{*}}$

and the abbreviation

$\eta(r)=1-\frac{1}{\sigma-1} \nabla^{*} \frac{r_{0}}{H_{\mathrm{p} 0}}\left(1-\left(\frac{r_{0}}{r}\right)^{\sigma-1}\right)$,

where $H_{\mathrm{p} 0}=p_{0} /\left(\rho_{0} g_{0}\right)$ is the pressure scale height at level $r_{0}$, we can write the solutions for the density and pressure stratification, respectively, in the form

$\rho(r)=\rho_{0} \eta(r)^{1 / \nabla^{*}-1} \quad$ and $\quad p(r)=p_{0} \eta(r)^{1 / \nabla^{*}}$.

The upper boundary of the polytropic layer, $R_{\mathrm{pl}}$, is defined by $\eta_{\mathrm{e}}\left(R_{\mathrm{pl}}\right)=0$.

We assume an isolated, toroidal flux tube with magnetic field strength $B_{0}$, located at $r=r_{0}$ in the equatorial plane. The flux ring is in mechanical equilibrium between the magnetic tension and buoyancy forces, viz.

$\frac{B_{0}^{2}}{4 \pi r_{0}}=\left(\rho_{\mathrm{e} 0}-\rho_{0}\right) g_{0}$,

where $\rho_{\mathrm{e} 0}$ and $\rho_{0}$ are the densities outside and inside the tube, respectively. The corresponding values of the pressure are related through the condition of total pressure balance,

$p_{\mathrm{e} 0}=p_{0}+\frac{B_{0}^{2}}{8 \pi}=p_{0}\left(1+\frac{1}{\beta_{0}}\right)$

with $\beta_{0}=8 \pi p_{0} / B_{0}^{2}$. As sketched in Fig. 7 , the tube is stretched into a semi-elliptical configuration in order to simulate the formation of a slowly rising flux loop. Assuming quasi-static and adiabatic stretching, the stratification inside the elongated tube is given by Eq. (7) with $\nabla^{*}=\nabla_{\mathrm{ad}}$, while the stratification of the surrounding convection zone is superadiabatic, $\nabla_{\mathrm{e}}^{*}=\nabla_{\mathrm{ad}}+\delta_{\mathrm{e}}$ (with

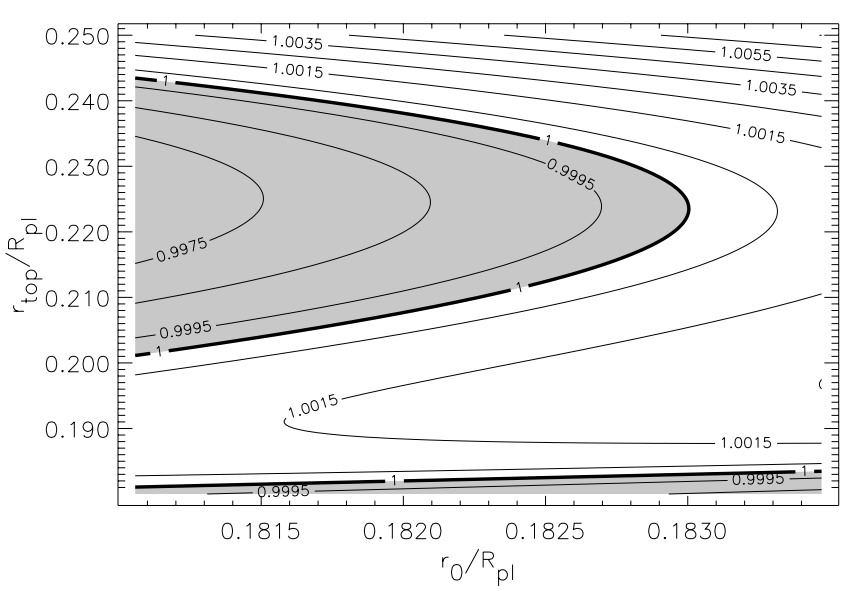

Fig. 8. Polytropic layer model: isolines of the acceleration ratio $\left|a_{\text {out }} / a_{\text {in }}\right|$ at the summit of the elongated flux tube, $r_{\text {top }}$, as a function of the initial radius of curvature, $r_{0} . R_{\mathrm{pl}}$ is the top of the polytropic layer $\left[\eta_{\mathrm{e}}\left(R_{\mathrm{pl}}\right)=0\right.$, Eq. (7)]. The domain where the magnetic tension force outweighs buoyancy is shaded grey. Thick lines represent states of (static) mechanical equilibrium.

$\left.\delta_{\mathrm{e}}>0\right)$. A quasi-static rise is a good approximation to the numerical results except for the uppermost, strongly superadiabatic layers of the convection zone. The base density, $\rho_{0}$, has to be adjusted as the loop is stretched to assure mass conservation within the tube. In order to decide whether the flux loop becomes trapped, we consider the ratio of the outward directed buoyancy acceleration, $a_{\text {out }}$, and the inward directed magnetic tension acceleration, $a_{\mathrm{in}}$, at the summit of the elongated part as a function of height. Figure 8 shows isolines of this ratio in the $\left(r_{0}, r_{\text {top }}\right)$-plane; the numerical values are chosen to resemble the conditions in the $1 M_{\odot}$ model No. 540: $\rho_{\mathrm{e} 0}=0.4 \mathrm{~g} / \mathrm{cm}^{3}, p_{\mathrm{e} 0}=$ $2.1 \times 10^{14} \mathrm{dyn} / \mathrm{cm}^{2}, g_{0}=2.3 \times 10^{3} \mathrm{~cm} / \mathrm{s}^{2}$ and $\beta_{0}=10^{6}$. The gravitational exponent is set to $\sigma=1.3$, taking into account that a significant fraction of the stellar mass is contained in the convection zone $\left(M\left(r_{0}\right) / M_{\star} \simeq 0.34\right)$. The adiabatic temperature gradient is $\nabla_{\text {ad }}=0.4$ and the superadiabaticity $\delta_{\mathrm{e}}=10^{-6}$. The equilibrium radius varies around $r_{0} \approx 4.28 \times 10^{10} \mathrm{~cm}$. For $r_{0} / R_{\mathrm{pl}} \lesssim 0.183$, Fig. 8 shows the existence of a domain (shaded grey) where the tension force is stronger than the buoyancy force. This region is bounded by two equilibrium states, a stable lower and an unstable upper state. Flux tubes reaching the lower state of equilibrium are trapped.

We compare this result with a similar representation of the non-linear simulations in a complete stellar model. Figure 9 shows the ratio of the outward and inward directed forces,

$\left|\frac{f_{\text {out }}}{f_{\text {in }}}\right|=\left|\frac{f_{\text {buoy }}+f_{\text {inertia }}}{f_{\text {curv }}+f_{\text {drag }}}\right|$,

at the summits of rising flux tubes starting between $25^{\circ}$ and $30^{\circ}$ latitude in the $1 M_{\odot}$ model No. 540. It also reveals a domain where the sum of the inward directed forces 


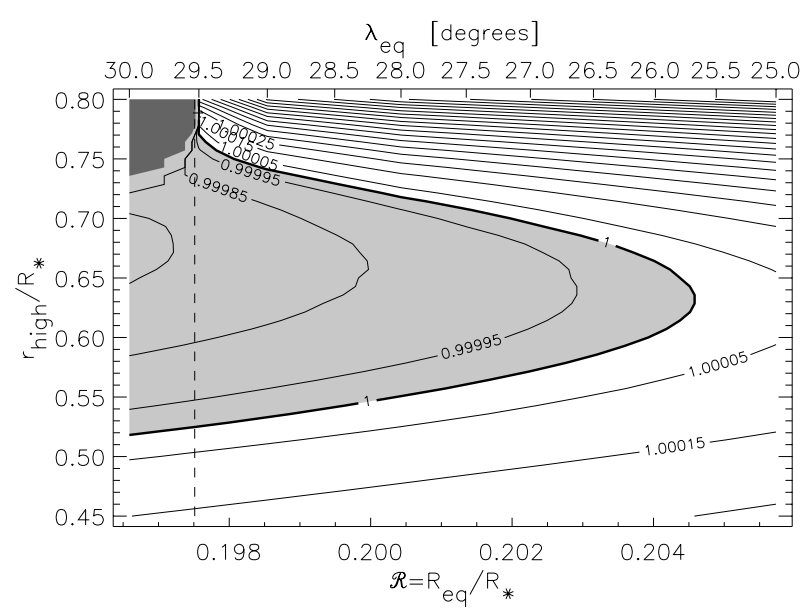

Fig. 9. Non-linear simulations in the $1 M_{\odot}$ model No. 540: isolines of the ratio $\left|f_{\text {out }} / f_{\text {in }}\right|$ at the summit of the rising flux loop, $r_{\text {high }}$, as a function of the initial relative radius of curvature, $\mathcal{R}=R_{\text {eq }} / R_{\star}$, (lower axis) and equilibrium latitude, $\lambda_{\text {eq }}$ (upper axis). The transition latitude is $\lambda_{\text {eq }} \approx 29.5^{\circ}$. The dark grey shaded region in the upper left corner contains no data, since trapped flux tubes beyond the transition line do not reach these heights.

dominates. The striking qualitative similarity of corresponding structures in both Figs. 8 and 9 confirms the analogy between the simplified and the full flux tube model $^{1}$. Tubes originating not far below the transition latitude experience an intermediate phase of deceleration during their rise in the middle of the convection zone. Owing to their inertia they traverse this region without becoming trapped. However, the smaller the initial radius of curvature, $\mathcal{R}$, the wider the decelerating region and, therefore, the larger the probability for a flux tube to be captured. Above the transition line, flux tubes do no longer rise beyond the decelerating region; they typically exceed their final equilibrium height until the retracting magnetic tension force pulls the summit back to the new stable equilibrium position (see trajectories starting right above the transition latitude in Fig. 4). For even smaller initial radii, the acceleration at small elongations is so weak that inertia effects become negligible and the flux tube asymptotically approaches its new equilibrium.

Since the results of the simple static model resemble these of the non-linear simulations remarkably well, the trapping mechanism indeed seems to be caused by the balance between magnetic tension and buoyancy forces.

\footnotetext{
1 The discrepancy between the relative location of the decelerating region inside the polytropic layer and convection zone, respectively, is due to the simplifications of the polytropic layer model. The polytropic model considers semi-elliptical elongated tubes which still enclose the radiative core, whereas in the simulation most of the tube resides in the convection zone of the star (cf. Fig. 3).
}

\section{Discussion}

\subsection{Toward the "Coronal Dividing Line" (CDL)}

The onset of complete trapping of magnetic flux tubes in models of low and moderate mass $\left(1 M_{\odot} \leq M_{\star} \leq\right.$ $\left.3 M_{\odot}\right)$ stars occurs in the temperature range $T_{\text {eff }} \simeq$ $4750 \ldots 4900$ K. Stellar models of similar evolutionary stage (i.e., with the same fractional size of the radiative core) calculated using the OPAL equation of state (Rogers et al. 1996) or using the Geneva-Toulouse evolution code (e.g., Charbonnel \& Talon 1999) are shifted by about $\left|\Delta T_{\text {eff }}\right| \lesssim 60 \mathrm{~K}$ in comparison to the evolutionary tracks described in Sect. 2. The transition from erupting to trapped flux tubes on the basis of these additional models is almost identical to our "standard" simulations and, therefore, the determined temperature range given above is quite robust. Using conversion tables (SchmidtKaler 1982), we find that the onset of flux tube trapping spans the spectral type range G7 to K0 of ordinary (luminosity class III) giant stars. However, it should be noted that we only considered "first-crossing" stars, i.e., stars which are on their first ascent along the RGB. At the tip of the RGB, stars with $M \lesssim 3 M_{\odot}$ start their Helium core burning phase with the Helium flash and then return into the region around the base of the giant branch for a second time, thus producing a heterogenous sample of stars with different internal structures known as the "K clump". These more evolved post-Helium-flash stars are not considered here. Nevertheless, we find the HR domain of trapped magnetic flux tubes to be situated very close to the left of the region where various sources in the literature locate the CDL, viz. K1...K3.

Emerging magnetic flux tubes lead to extended bipolar regions in stellar atmospheres, providing large loops capable of confining hot $\left(T>10^{5} \ldots 10^{6} \mathrm{~K}\right)$ transition region and coronal plasma. This is crucial because the small gravity in the atmosphere of giant stars is not capable to retain hot coronal matter. Since the transition region DL (Linsky \& Haisch 1979) and coronal DL (Ayres et al. 1981) coincided with the line in the HR diagram which marks the onset of substantial mass-loss by cool and slow stellar winds (Reimers 1977), it was suggested - among other scenarios - that the reason for the fading of coronal $\mathrm{X}$-ray emission is a change in magnetic field structure.

Rosner et al. (1991, 1995) and Kashyap et al. (1994) assume a change in the topology of the atmospheric magnetic field from large, coherent coronal loops (to the hot side of the CDL) to an open field line geometry with only small loops remaining (to the cool side). The larger surface fraction covered by open field lines then allows for the escape of a cool, massive stellar wind which is thought to be driven by Alfvén waves. As a reason for this transition a change in dynamo nature is suggested: from a solar-like dynamo producing large-scale, toroidal magnetic fields to a dynamo operating on the small spatial scales of the turbulent flow field, which still can maintain low-level magnetic and X-ray activity. 
The trapping mechanism found in our study supports this scenario, since it provides a physical reason for the transition of field topology, even without invoking a change of the dynamo mode. Furthermore, the emergence of flux elements "shredded" from trapped loops by convection may also contribute to low-level activity. The change from large-scale to small-scale loops could also be related to the proposal of Ayres et al. (1998), who discuss the possibility of small, submerged coronal loops attenuated by the "circumcoronal" environment. Antiochos et al. (1986) found that hot coronal loops may become thermally unstable due to the low surface gravity in giant stars; the plasma tends to a stable, cooler configuration and emits its energy at longer wavelengths. In the light of our calculations, this effect could, in principle, occur in stars before the onset of flux tube trapping. However, stars in the HR region to the left of the trapping domain show strong X-ray emission. Hünsch \& Schröder (1996) questioned the original concept of a dividing line and argued that the distribution of X-ray active giants can be understood as a consequence of the dependence of rotational history on stellar mass, without requiring a quasi-discontinuous change of stellar activity along the evolutionary track. They argue that low-mass stars $\left(M \lesssim 1.3 M_{\odot}\right)$ are strongly spun down by magnetic braking during their long main-sequence phase, so that they become essentially non-active when becoming giants. Higher-mass stars, on the other hand, develop an outer convection zone not before they cross the Hertzsprung gap, so that they retain enough angular momentum to maintain dynamo action and X-ray activity when they arrive at the base of the RGB. The CDL would then become just the evolutionary path of a $1.3 M_{\odot}$ star, representing the transition between stars with and without outer convection zones on the main sequence. On the other hand, our results predict a rather abrupt change in the surface activity in the course of the evolution of a giant, which leads to a corresponding strong decrease of its X-ray emission. The trapping of magnetic flux is practically independent of rotation rate and should therefore occur in all giants of appropriate mass and evolutionary stage as long as a dynamo mechanism provides magnetic fields. A decision between this view of the CDL and the model of Hünsch \& Schröder (1996) could come from more sensitive X-ray observations of a large number of giants together with the calculation of reliable evolutionary tracks in order to determine whether the tracks cross or move parallel to the CDL.

\subsection{Model restrictions and consequences}

The most radical restriction in this work is the use of the thin-flux-tube approximation for the description of the magnetic field inside a star. It receives its legitimation from the good agreement of flux tube simulations with observations of the properties of bipolar magnetic regions in the case of the sun (Caligari et al. 1995). The very existence of sunspots and the whole hierarchy of smaller flux elements as strong flux concentrations embedded in a much less magnetized environment indicates that stellar magneto-convection tends to form such structures that lend themselves to a simplified mathematical representation in terms of the thin-flux-tube approximation. We have no reason to assume that the convection zone of a giant would behave fundamentally different in this respect.

On the other hand, since the treatment of the full MHD-problem over the whole range of the convection zone is far beyond current computational capabilities, we cannot demonstrate the formation process of the magnetic flux tubes from first principles, so that our initial conditions are somewhat arbitrary. The same is true, of course, for the generation of the magnetic flux by a dynamo mechanism in the first place. Hence, we must keep in mind that our calculations can only represent a crude approximation to the situation inside a real convection zone, where the magnetic field is mingled with turbulent convective motions. However, the case of the Sun, where a detailed comparison with observations is possible, shows that the flux tube model catches the essential dynamics of strongfield magnetic structures in the convection zone, lending some credibility to our approach.

Structure and extent of a stellar overshoot region are not well understood. In the case of the Sun, analysis of helioseismic data (e.g., Basu 1997) indicates a much smaller depth of the overshoot region than predicted by theoretical models, albeit under the (probably unrealistic) assumption of a perfectly spherically symmetric overshoot region. The important quantities for the flux tube model are the width and the subadiabaticity of the overshoot layer: the layer must be sufficiently thick to store the necessary amount of magnetic flux while the value of the subadiabaticity determines the critical field strength for the undulatory instability. We have carried out flux tube simulations based upon stellar models generated with the Geneva-Toulouse stellar evolution code (e.g., Charbonnel \& Talon 1999) and the overshoot model of Zahn (1991) and found that evolution and trapping of flux tubes in these stellar models are virtually identical to the results given here (Schüssler et al. 2001). This is not surprising since, as we have shown above, the trapping mechanism is dominated by the forces acting on the crest of the loop, which is located within the convection zone. Likewise, the trapping is not sensitively dependent on the initial depth of the equilibrium, which corresponds to a variation of the subadiabaticity. We conclude that our principal results are not strongly affected by the uncertainties concerning the structure of stellar overshoot regions, as long as a sufficiently thick overshoot region exists at all.

Our simulations include neither convective motions nor differential rotation and meridional flows. Although such motions certainly exist, the high value of the threshold for the undulatory instability leads to a magnetic energy density of the flux tubes exceeding the kinetic energy of these motions (super-equipartition magnetic fields). Once inside the convection zone proper, the dynamics of a rising loop is dominated by convective and magnetic buoyancy, 
so that external motions do not strongly affect the evolution of the loop. Likewise, solar-type differential rotation inside the overshoot region does hardly change the stability properties (Ferriz-Mas \& Schüssler 1995). Of course, in the absence of conclusive observational evidence and reliable theoretical models, we cannot exclude exotic situations with an extremely strong differential rotation in the interior of a giant, which could lead to more profound changes in the dynamics of rising flux tubes.

All investigated flux tubes originate from the same typical stage, i.e., a toroidal flux ring in mechanical equilibrium in the overshoot region parallel to the equatorial plane. Although this seems a reasonable assumption - in view of an (supposed) $\alpha \Omega$-dynamo operating in the lower regions of the outer convection zone and the need to store the magnetic field - we cannot rule out the possible existence of other equilibria which could lead to different results.

The neglect of flux tube interactions may become unrealistic in view of the pole- and equatorward slip of an anchored tube segment inside the overshoot region, where we would expect them to meet with other stored tubes. These interactions probably complicate the downward drift of trapped tubes and may affect the evolution of the loop summit. Similarly, it is unlikely that a trapped flux tube drifts down toward the equator in the continuous, steady manner described in Sect. 4, and, since the time to reach the new equilibrium is rather long, it may not reach this state at all. Anyway, whether a trapped flux tube finds a new equilibrium in the equatorial plane, becomes "shredded" by convective motions, or reconnects with other magnetic fields, the important result is the lack of emergence of coherent magnetic field at the stellar surface. The trapping mechanism does probably not lead to a complete switchoff of magnetic activity, but it will cause a significant drop of X-ray emission among cool giants.

Our parameter study has shown that the trapping mechanism is quite robust, but further aspects have still to be considered: does flux tube trapping also occur in higher-mass stars (hybrid stars), more evolved "K clump" giants, and evolved members of fast-rotating, active RS CVn binaries? Young pre-main-sequence stars also possess small radiative cores and exhibit magnetic activity. Is a trapping mechanism there possible, too? Furthermore, future work should include interactions between flux tubes and large-scale flows.

\section{Conclusion}

The extension of the flux tube model to post-mainsequence giants of low and moderate mass reveals a trapping mechanism for magnetic flux tubes, which sets in when evolving stars reach the HR domain of the "Coronal Dividing Line". A force analysis shows that the increase of curvature at the summit of a rising magnetic loop leads to the dominance of the magnetic tension force over the buoyancy force when the relative radius of curvature of the initial equilibrium flux ring falls short of the critical value $\mathcal{R}=R_{\text {eq }} / R_{\star}=0.17 \ldots 0.2$, thus leading to the termination of a loop's ascent inside the convection zone. A parameter study shows that the trapping mechanism is quite robust and practically independent of the detailed stellar structure and other parameters like magnetic field strength and rotation rate; the fate of a rising flux loop can be predicted solely by the value of $\mathcal{R}$ of its parent equilibrium flux ring. Flux tube trapping leads to the vanishing of large-scale active regions and coronal loops and thus is in accordance with the proposal of Rosner et al. (1991, 1995) of a transition in magnetic field topology from large loops to the left of the CDL to a configuration dominated by open field lines and smaller loops to the right.

Acknowledgements. The authors are very grateful to Thomas Granzer for providing the stellar evolution code and his implementation of the non-local convection zone treatment, and to Corinne Charbonnel for providing stellar models generated with the Geneva-Toulouse evolution code. Sami K. Solanki contributed to this work with many helpful discussions and much support.

\section{References}

Alexander, D. R., \& Ferguson, J. W. 1994, ApJ, 437, 879

Antiochos, S. K., Haisch, B. M., \& Stern, R. A. 1986, ApJ, 307, L55

Ayres, T. R., Fleming, T. A., \& Schmitt, J. H. M. M. 1991, ApJ, 376, L45

Ayres, T. R., Linsky, J. L., Vaiana, G. S., Golub, L., \& Rosner, R. 1981, ApJ, 250, 293

Ayres, T. R., Simon, T., Stern, R. A., et al. 1998, ApJ, 496, 428

Basu, S. 1997, MNRAS, 288, 572

Caligari, P., Moreno-Insertis, F., \& Schüssler, M. 1995, ApJ, 441,886

Caughlan, G. R., \& Fowler, W. A. 1988, Atom. Nucl. Data Tab., 40, 283

Charbonnel, C., \& Talon, S. 1999, A\&A, 351, 635

Ferriz-Mas, A., \& Schüssler, M. 1993, Geophys. Astrophys. Fluid Dyn., 72, 209

Ferriz-Mas, A., \& Schüssler, M. 1995, Geophys. Astrophys. Fluid Dyn., 81, 233

Granzer, T., Schüssler, M., Caligari, P., \& Strassmeier, K. G. 2000, A\&A, 355, 1087

Haisch, B., Schmitt, J. H. M. M., \& Rosso, C. 1991, ApJ, 383, L15

Haisch, B. M., Bookbinder, J. A., Maggio, A., Vaiana, G. S., \& Bennett, J. O. 1990, ApJ, 361, 570

Hünsch, M., Schmitt, J. H. M. M., Schröder, K., \& Reimers, D. 1996, A\&A, 310, 801

Hünsch, M., Schmitt, J. H. M. M., Schröder, K., \& Zickgraf, F. 1998, A\&A, 330, 225

Hünsch, M., \& Schröder, K. 1996, A\&A, 309, L51

Iglesias, C. A., \& Rogers, F. J. 1996, ApJ, 464, 943

Kashyap, V., Rosner, R., Harnden, F. R., et al. 1994, ApJ, 431, 402 
Kippenhahn, R., Weigert, A., \& Hofmeister, E. 1967, Meth. Comp. Phys., 7, 129

Linsky, J. L., \& Haisch, B. M. 1979, ApJ, 229, L27

Maggio, A., Vaiana, G. S., Haisch, B. M., et al. 1990, ApJ, 348, 253

Moreno-Insertis, F., Schüssler, M., \& Ferriz-Mas, A. 1992, A\&A, 264, 686

Pidatella, R. M., \& Stix, M. 1986, A\&A, 157, 338

Reimers, D. 1977, A\&A, 57, 395

Reimers, D., Hünsch, M., Schmitt, J. H. M. M., \& Toussaint, F. 1996, A\&A, 310, 813

Reimers, D., \& Schmitt, J. H. M. M. 1992, ApJ, 392, L55

Rogers, F. J., Swenson, F. J., \& Iglesias, C. A. 1996, ApJ, 456, 902

Rosner, R., An, C., Musielak, Z. E., Moore, R. L., \& Suess, S. T. 1991, ApJ, 372, L91

Rosner, R., Musielak, Z. E., Cattaneo, F., Moore, R. L., \& Suess, S. T. 1995, ApJ, 442, L25
Schmidt-Kaler, T. 1982, Landolt-Börnstein - New Series, Grp.VI, vol. 2, Astronomy and Astrophysics, ed. K. Schaifers, \& H. H. Voigt (Springer-Verlag), 456

Schüssler, M., Caligari, P., Ferriz-Mas, A., \& Moreno-Insertis, F. 1994, A\&A, 281, L69

Schüssler, M., Caligari, P., Ferriz-Mas, A., Solanki, S. K., \& Stix, M. 1996, A\&A, 314, 503

Schüssler, M., Holzwarth, V., Charbonnel, C., \& Solanki, S. K. 2001, in ASP Conf. Ser. 223, 11th Cambridge workshop on cool stars, Stellar systems and the Sun, ed. R. J. García López, R. Rebolo, \& M. R. Zapatero Osorio, CD-1114

Schüssler, M., \& Solanki, S. K. 1992, A\&A, 264, L13

Shaviv, G., \& Salpeter, E. E. 1973, ApJ, 184, 191

Skaley, D., \& Stix, M. 1991, A\&A, 241, 227

Spruit, H. C. 1981, A\&A, 102, 129

Spruit, H. C., \& van Ballegooijen, A. A. 1982, A\&A, 106, 58

Strassmeier, K. G. 1999, A\&A, 347, 225

Zahn, J.-P. 1991, A\&A, 252, 179 